\title{
Reaching key populations with community-based HIV test and start services in Lagos and Benue States in Nigeria: Baseline findings
}

Project SOAR

Follow this and additional works at: https://knowledgecommons.popcouncil.org/departments_sbsr-hiv

Part of the Community-Based Research Commons, and the International Public Health Commons How does access to this work benefit you? Let us know!

\section{Recommended Citation}

Project SOAR. 2019. "Reaching key populations with community-based HIV test and start services in Lagos and Benue States in Nigeria: Baseline findings," Results brief. Washington, DC: Population Council. 


\section{Reaching Key Populations with Community-based HIV Test and Start Services in Lagos and Benue States in Nigeria: baseline findings}

Key populations (KPs), such as men who have sex with men (MSM), female sex workers (FSWs), and people who inject drugs, remain disproportionately affected by HIV compared to the general population. In Nigeria, HIV prevalence has declined among the general population (3.6 to 1.4 percent) from 2007 to 2018. ${ }^{1}$ Among key populations from 2007 to 2014 , HIV also decreased among FSWs (brothel based-37 to 19 percent; non-brothel based-30 to 9 percent); but increased among MSM (14 to 23 percent). ${ }^{2}$ Evidence-based strategies to sustain the decline among the general population and FSWs, and mitigate the increase among MSM, are needed.

Nigeria has adopted the World Health Organization's "test and start" (TnS) strategy, which recommends initiating antiretroviral therapy (ART) by persons living with HIV as soon as possible, irrespective of their CD4 cell count. ${ }^{3}$ Community-based TnS service delivery is a promising strategy for increasing access to testing, treatment, and care for KPs.

In Nigeria, community-based HIV services for KPs are offered through one-stop shops to meet their comprehensive prevention, treatment, and care needs. One-stop shops are "safe spaces" where KPs receive non-discriminatory services. They require less time and travel expenses than secondary and tertiary health institutions, minimize stigma, and promote quality care in a culturally sensitive manner.

Project SOAR in partnership with the Federal Ministry of Health (FMoH), National Agency for the Control of AIDS (NACA), U.S. President's Emergency Plan for AIDS Relief (PEPFAR), and U.S. Agency for International Development (USAID), are implementing a prospective cohort study to assess the effectiveness of community-based TnS models in reaching HIV-positive MSM and FSWs, retaining them on ART, and achieving viral suppression. The study is being conducted in Lagos and Benue states.

This brief highlights findings from the baseline survey of MSM and FSWs who tested positive at enrollment.

\section{Description of community- based, service delivery models}

\section{Lagos State}

The three study clinics (Yaba, Agege, and Badagry) are one-stop shops operated by full-time clinical teams that provide comprehensive HIV prevention, care and treatment services. They also provide peer education, condoms and lubricants, STI management, HIV testing services, laboratory services, prevention messaging, ART, and care and psychosocial support. The Yaba one-stop shop is run by the Population Council and the other two by Heartland Alliance.

\section{Benue State}

The two study sites (Gboko and Markudi) are drop-in centers where HIV prevention services for MSM and FSWs are provided by Heartland Alliance, while ART services and care are provided by a PEPFAR treatment partner. Complex cases are referred to the treatment partner-supported facilities.

\section{RECRUITMENT}

Between December 2017 and June 2018 we used community mobilizers to recruit study participants through outreach. We also approached eligible clients walking into the study clinics. Outreach services were led by MSM and FSW community mobilizers who promoted HIV testing and generated awareness of HIV testing events among their peers at culturally appropriate outreach spots. At HIV testing events, one-stop shops were promoted to increase awareness of their presence and services. 


\section{RESULTS}

A total of 605 treatment naïve participants living with HIV were enrolled in the study and participated in the baseline survey (340 in Lagos and 265 in Benue; Figure 1).

Figure 1 Key population distribution by state Lagos

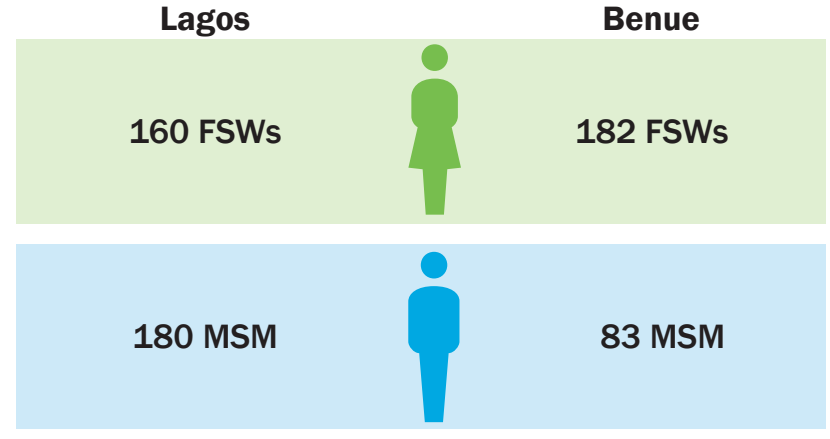

More than half of MSM were walk-ins while the vast majority of FSWs were recruited by outreach (Figure 2).

Figure 2 Recruitment distribution by population
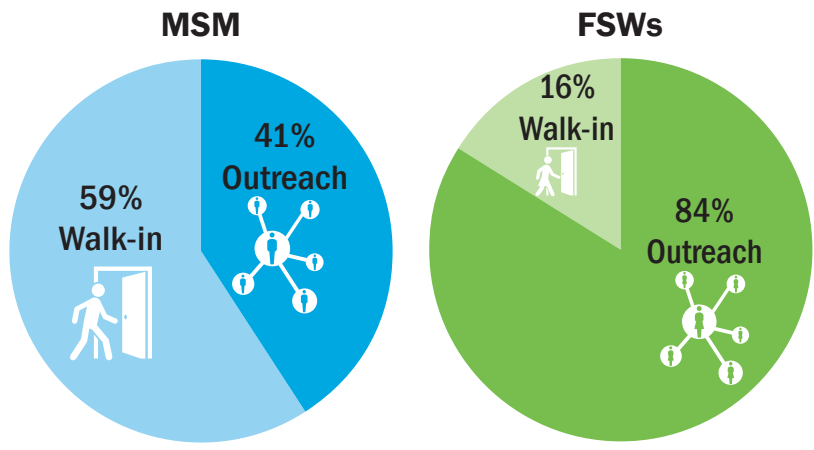

\section{How did participants vary by type of recruitment?}

\section{MSM}

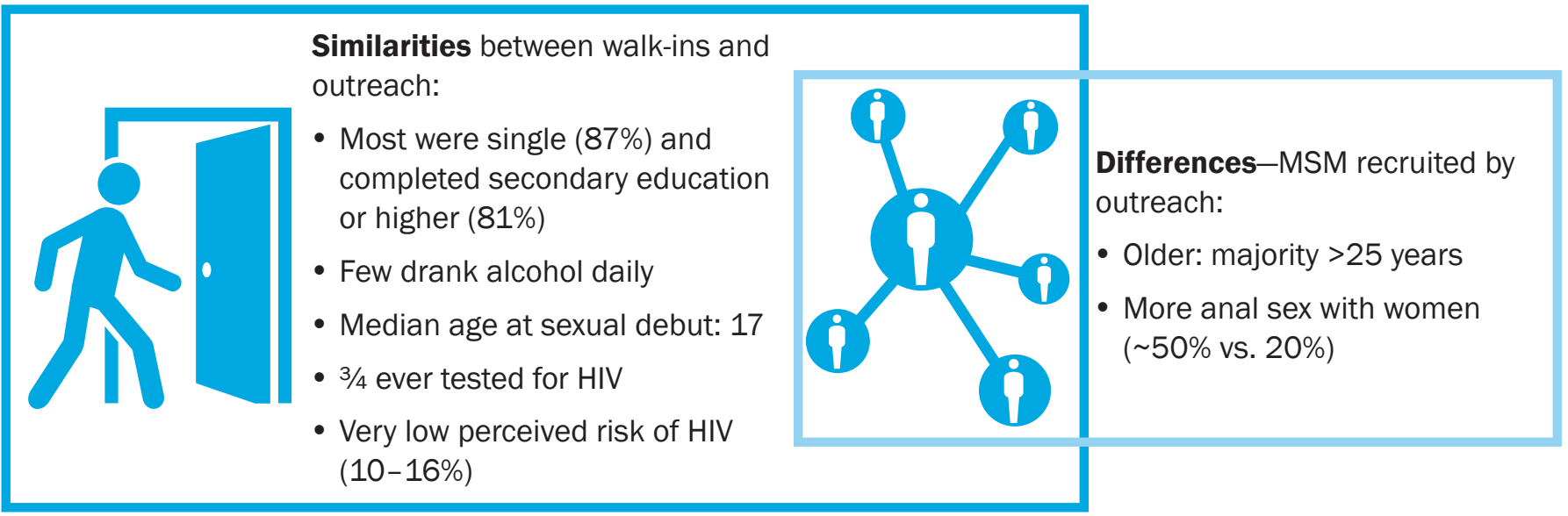

\section{FSWs}

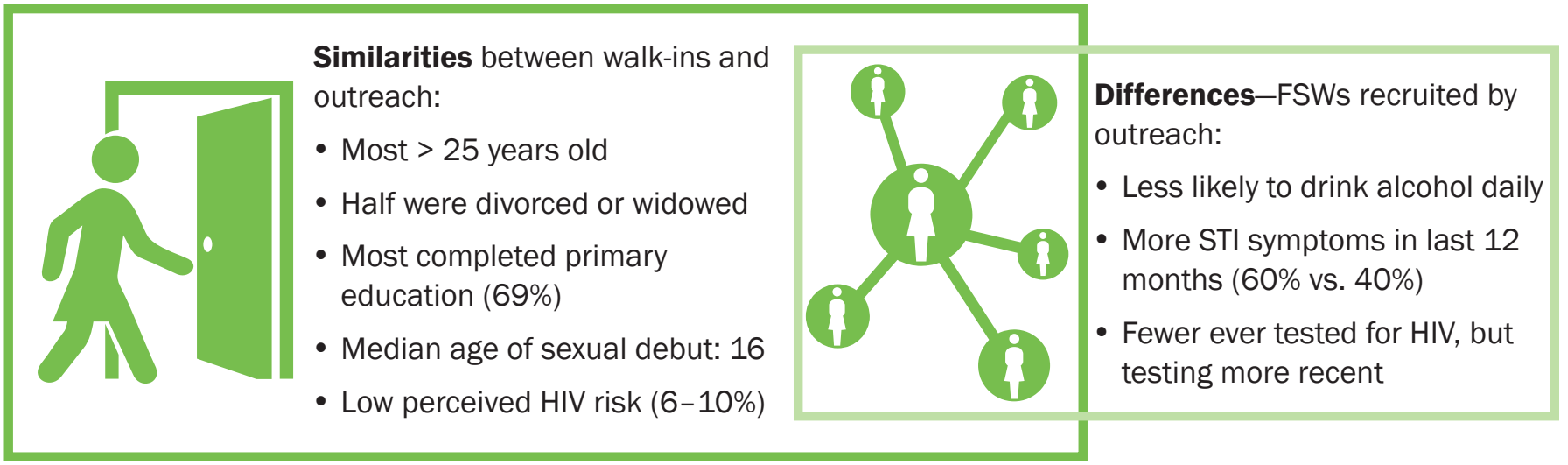

2 | Reaching key populations with community-based HIV TnS services in Lagos and Benue States in Nigeria: baseline findings 
Recent HIV testing is more common among MSM and FSWs recruited through outreach

Figure 3 HIV testing within past 3 months by type of recruitment

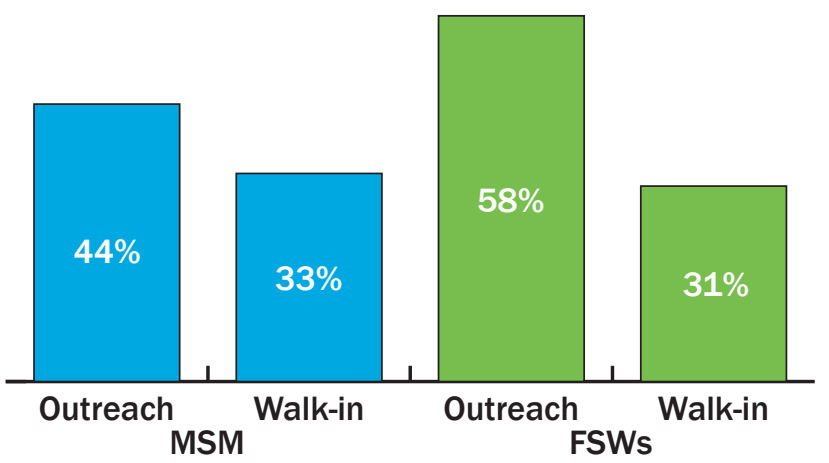

STI symptoms and treatment among MSM and FSWs varies by mode of recruitment.

- More MSM walk-ins reported an STI symptom in the last 12 months and were treated for last STI.

- Fewer FSW walk-ins reported an STI symptom but more likely to be treated.

Figure 4 STI symptoms and treatment by type of recruitment

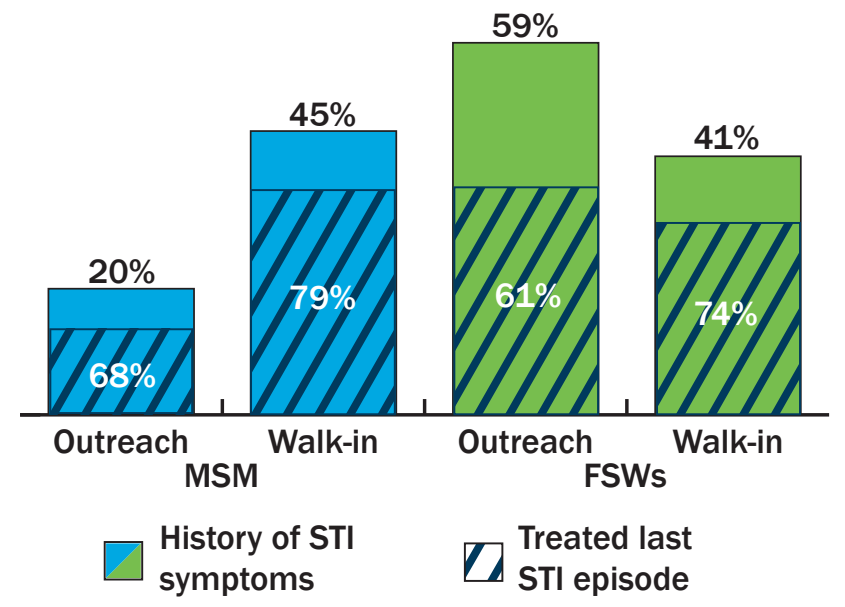

\section{How did participants vary by state?}

MSM in Lagos and Benue reported varying risky sexual behaviors.

MSM in Lagos had:

- Less anal sex with women (1 in 5 vs. 3 in 4)

- More lubricant use (2x as many)

- More receptive anal sex (1 in 3 vs. 1 in 20)

\section{Ever tested for HIV was more common among MSM in Benue than Lagos.}

Figure 5 Ever tested for HIV among MSM by state

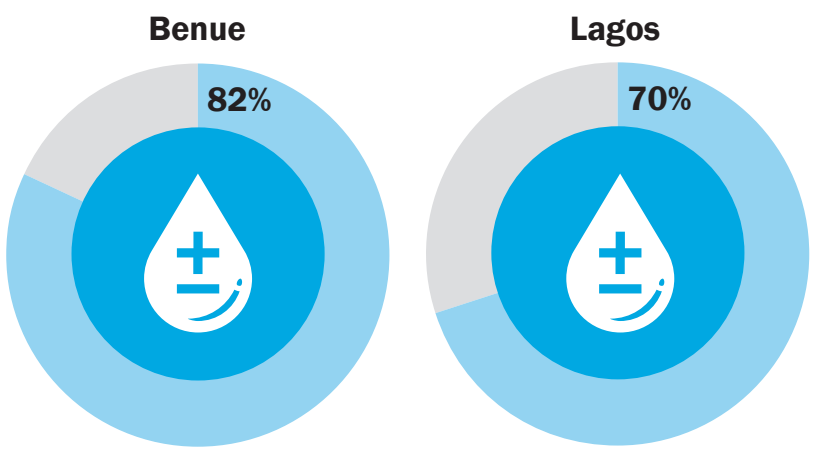

\section{Recent HIV testing was more common among MSM and FSW in Benue than Lagos.}

Figure 6 Recent HIV testing among MSM and FSWs by state

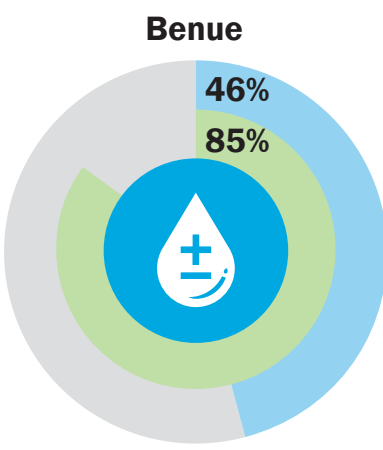

MSM

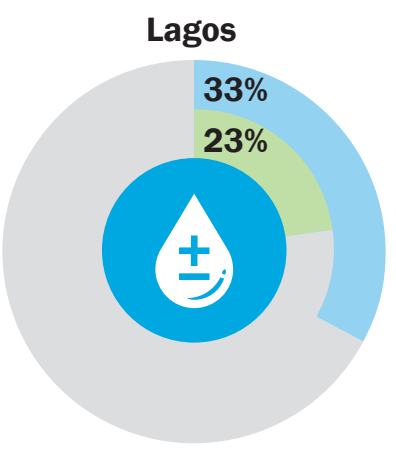

FSWs 


\section{More MSM in Lagos reported a recent STI symptom and were more likely to be treated.}

Figure 7 STI symptoms and treatment among MSM by state

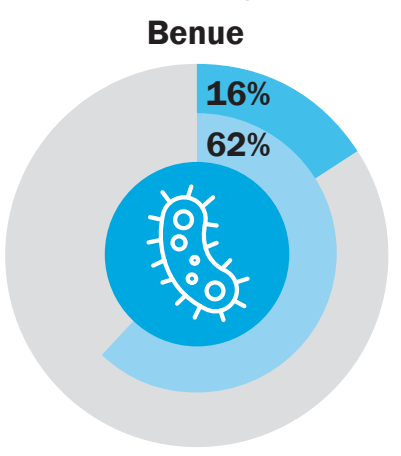

STI symptom in last 12 months

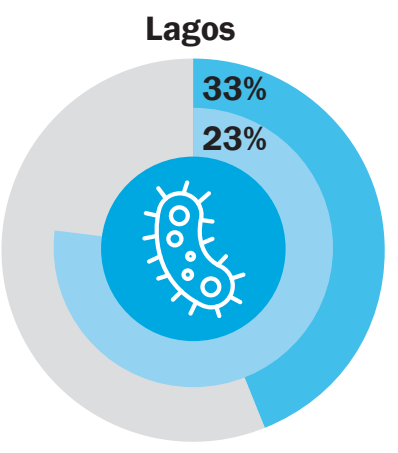

Treated for STI

\section{More FSWs in Benue reported a recent STI symptom, and were less likely to be treated.}

\section{Figure 8 STI symptoms and treatment among FSWs by state}

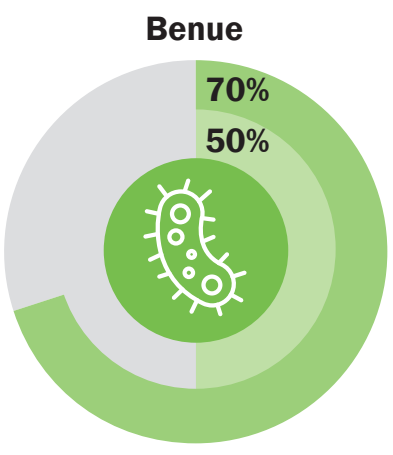

STI symptom in last 12 months

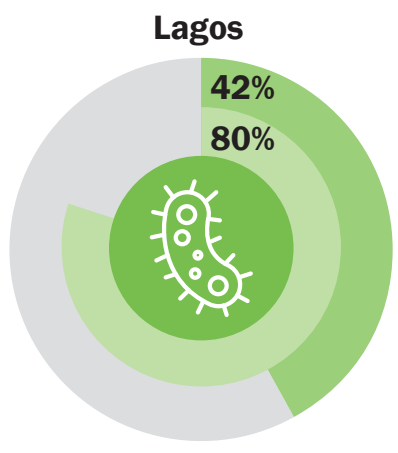

Treated for STI

\section{CONCLUSION}

MSM and FSWs recruited for this study through outreach and by approaching walk-in clients presented different demographic and sexual risk profiles at baseline. Having accessible clinics to accommodate walk-ins, as well as conducting outreach for prevention and HIV testing for those

Project SOAR is a six-year (September 2014-September 2020) cooperative agreement funded by the U. S. President's Emergency Plan for AIDS Relief and the U. S. Agency for International Development (Agreement No. AIDOAA-A-14-00060). The contents of this brief are the sole responsibility of Project SOAR and Population Council and do not necessarily reflect the views of PEPFAR, USAID, or the United States Government.

at high risk of HIV, are both important for reaching these two populations in the study sites.

There appeared to be some state-level differences among MSM and FSWs enrolled in the study as well. Risky sexual behaviors among MSM in Benue included high rates of anal sex with women and lower use of lubricants during sex. In Lagos, more men reported receptive-only sex than in Benue. Differences in engagement with HIV services were also evident. MSM in Lagos were more likely to have been treated for an STI while MSM in Benue were more likely to have been previously tested for HIV. Finally, FSWs in Benue appeared to have had slightly less engagement with HIV services than in Lagos.

Although nearly three-fourths of MSM and FSWs in the study overall had ever been tested for HIV, less than 60 percent had been tested in the last three months, which is the national recommendation for KPs. Additionally, few MSM and FSWs felt at risk to HIV despite their risk behaviors. This suggests that messages for KPs need to be better tailored to enhance risk perception and the importance of HIV testing. There is also a need to examine whether novel approaches, such as HIV self-testing, increase the frequency of testing among KPs. Finally, the study highlights the importance of making highquality, KP-friendly services readily available.

Endline study results will provide important data on the effectiveness of the two community-based HIV test and start service delivery models in Lagos and Benue.

\section{REFERENCES}

${ }^{1}$ National Agency for the Control of AIDS. 2019. "Nigeria HIV/AIDS Indicator and Impact Survey national summary sheet." https://naca. gov.ng/naiis-national-summary-sheet/. Accessed August 2019

${ }^{2}$ National HIV/AIDS \& STIs Control Programme Federal Ministry of Health, Nigeria. 2015. Integrated Biological and Behavioural Surveillance Survey (IBBSS) 2014. Abuja: FMoH, Nigeria.

${ }^{3}$ World Health Organization. 2015. Guideline on when to start antiretroviral therapy and on pre-exposure prophylaxis for HIV. Geneva: World Health Organization.

Suggested citation: Project SOAR. 2019. "Reaching key populations with community-based HIV test and start services in Lagos and Benue States in Nigeria: baseline findings," Project SOAR Results Brief. Washington, DC: Population Council.
Project SOAR/Population Council

4301 Connecticut Avenue, NW, Suite 280

Washington, DC 20008

Tel: +1202 2379400

e-mail: ProjectSOAR@popcouncil.org projsoar.org

(C) Population Council, August 2019 